IJMMS 31:1 (2002) 11-21

PII. S0161171202110337

http://ijmms.hindawi.com

(c) Hindawi Publishing Corp.

\title{
THE HAUSDORFF DIMENSION AND EXACT HAUSDORFF MEASURE OF RANDOM RECURSIVE SETS WITH OVERLAPPING
}

\author{
HONGWEN GUO and DIHE HU
}

Received 15 October 2001

\begin{abstract}
We weaken the open set condition and define a finite intersection property in the construction of the random recursive sets. We prove that this larger class of random sets are fractals in the sense of Taylor, and give conditions when these sets have positive and finite Hausdorff measures, which in certain extent generalize some of the known results, about random recursive fractals.
\end{abstract}

2000 Mathematics Subject Classification: 60D05, 28A80.

1. Introduction. As it is known, the separation conditions, such as the strong separation condition, the open set condition (OSC), and the strong open set condition, must be taken into consideration when computing the Hausdorff dimensions of the random recursive sets. In deterministic cases, Schief [13] proved that the strong open set condition and the open set condition are both equivalent to $\infty>\mathscr{H}^{\alpha}(K)>0$, where $K$ is the strictly self-similar set (cf. Hutchinson [9]) in $\mathbb{R}^{d}, \alpha$ is the similarity dimension of $K$, and $\mathscr{H}^{\alpha}$ denotes the Hausdorff measure of this dimension. But in random cases, we do not have such good results, many authors, such as Cawley and Mauldin [2], Falconer [3], Graf [6], Mauldin and Williams [12], Arbeiter and Patzschke [1], and $\mathrm{Hu}[7,8]$, have discussed the fractal properties of the random recursive set $K(\omega)$, and the most general result may be: if the open set condition is satisfied in the random recursive process of i.i.d. contraction similitudes, then $\operatorname{dim} K(\omega)=\alpha$ with probability one, where $\alpha$ is the unique solution of the equation

$$
\mathbf{E} \sum_{i=1}^{N} r_{i}^{\alpha}=1,
$$

and $\mathbf{E}$ is the expectation operator and $r_{i}$ is the Lipschitz coefficients of the similitudes.

Sometimes the open set condition in the construction of recursive sets is complex and difficult to verify. In this paper, we try to find another criterion to calculate the fractal dimensions of some random recursive sets, we give a definition of the finite intersection property (FIP) which allows appropriate overlapping in the same level. This condition is rather easy to verify, especially in the generalized Moran sets and Mauldin-Williams (M-W) models [12] (in fact, the open set condition is equivalent to the nonoverlapping in the same level in the recursive process of $\mathrm{M}-\mathrm{W}$ models). We prove that if the recursive process satisfies the OSC, then it satisfies the FIP, and we give examples which satisfy FIP but do not satisfy OSC; we also prove the following theorem. 
THEOREM 1.1. For every $\epsilon>0$, if $\left\{\left(S_{\sigma * 1}, \ldots, S_{\sigma * N}\right), \sigma \in D\right\} \subset \operatorname{sicon}\left(\Omega, \mathbb{R}^{d}\right)^{N}$ satisfies $\epsilon$-FIP, and $\left\{\left(r_{\sigma * 1}, \ldots, r_{\sigma * N}\right), \sigma \in D\right\}$ is a family of i.i.d. random elements, then

$$
\operatorname{Dim} K(\omega)=\operatorname{dim} K(\omega)=\min \{\alpha, d\} \quad \text { a.e. }
$$

still holds; furthermore if $\left\{\left(S_{\sigma * 1}, \ldots, S_{\sigma * N}\right), \sigma \in D\right\} \subset \operatorname{sicon}\left(\Omega, \mathbb{R}^{d}\right)^{N}$ satisfies 0-FIP, if $\sum_{i=1}^{N} r_{i}^{\alpha}=1$ a.e. and $\alpha \leq d$, then $\infty>\mathscr{H}^{\alpha}(K)>0$ a.e., where $\alpha$ is defined by (1.1), dim and Dim stand for Hausdorff dimension and Packing dimension, respectively, and $\mathscr{H}^{\alpha}$ is the Hausdorff measure.

2. Preliminaries. Let $(\Omega, \mathscr{F}, P)$ be a complete probability space, and let $(E, \rho)$ be a separable complete metric space. For all $f: E \mapsto E$, we call

$$
\operatorname{Lip}(f)=\sup _{x \neq y, x, y \in \mathbf{E}} \frac{\rho(f(x), f(y))}{\rho(x, y)}
$$

the Lipschitz coefficient of $f$. Denote

$$
\operatorname{con}(E)=\{f: \operatorname{Lip}(f)<1, f: E \longmapsto E\}
$$

In this paper, we take $E$ to be $\mathbb{R}^{d}$, the Euclidean space. Let $\operatorname{con}\left(\mathbb{R}^{d}\right)$ be equipped with the usual topology of uniform convergence on compact sets. Let $\operatorname{sicon}\left(\mathbb{R}^{d}\right)$ be the space of all contraction similitudes, and let $\operatorname{sicon}\left(\Omega, \mathbb{R}^{d}\right)$ be the space of all random contraction similitudes.

Let $N$ be a positive integer and let $C_{n}=\{1, \ldots, N\}^{n}$. Denote by $D=\bigcup_{n=0}^{\infty} C_{n}$ the family of all finite sequences $\sigma=\left(\sigma_{1}, \ldots, \sigma_{n}\right)$ in $\{1, \ldots, N\}$, by $|\sigma|=n$ the length of $\sigma \in C_{n}$, by $\tau \mid n=\left(\tau_{1}, \ldots, \tau_{n \wedge|\tau|}\right)$ the curtailment for $\tau \in D$, by $\sigma * \tau=\left(\sigma_{1}, \ldots, \sigma_{|\sigma|}, \tau_{1}, \ldots, \tau_{|\tau|}\right)$ the juxtaposition of $\sigma$ and $\tau$, by $C$ the infinite sequences $\sigma=\left(\sigma_{1}, \sigma_{2}, \ldots\right)$, and by $[\sigma]=\{\tau \in C, \tau|| \sigma \mid=\sigma\}$ the cylinder in $C$. For brevity, write $S_{(|\sigma|, \sigma)}=S_{\sigma \mid 1} \circ \cdots \circ S_{\sigma}$, $S_{(0, \varnothing)}=I$, where $S_{\tau} \in \operatorname{sicon}\left(\Omega, \mathbb{R}^{d}\right)$ for $\tau \in D$, and $I$ is the identity; $r_{\sigma}=\operatorname{Lip} S_{\sigma}$ and $\mathbf{r}_{(|\sigma|, \sigma)}=r_{\sigma \mid 1} \cdots r_{\sigma}$.

Throughout the paper, we suppose that $r \hat{=} \min _{\sigma \in C_{n}, n \geq 1} \operatorname{essinf} \operatorname{Lip}\left(S_{\sigma}\right)>0$ and $\max _{\sigma \in C_{n}, n \geq 1}$ ess $\sup \operatorname{Lip}\left(S_{\sigma}\right)<1$, and $E$ is a fixed nonempty compact subset of $\mathbb{R}^{d}$. For a set $J \subset E$, let $J^{0}$ denote the set of interior points of $J$.

DEFINITION 2.1. Let $\left\{\left(S_{\sigma * 1}, \ldots, S_{\sigma * N}\right), \sigma \in D\right\}$ be a collection of random elements from $(\Omega, \mathscr{F}, P)$ to $\operatorname{con}\left(\mathbb{R}^{d}\right)^{N}$. If there exists a nonempty open set $O$ in $E$, such that for $P$-a.e. $\omega$,

(i) $S_{(|\sigma|+1, \sigma * i)}(O) \subset S_{(|\sigma|, \sigma)}(O)$, for all $i=1, \ldots, N, \sigma \in D$.

(ii) $S_{(|\sigma|+1, \sigma * i)}(O) \cap S_{(|\sigma|+1, \sigma * j)}(O)=\varnothing$ for all $i \neq j, \sigma \in D$.

Then the family of $\left\{\left(S_{\sigma * 1}, \ldots, S_{\sigma * N}\right), \sigma \in D\right\}$ satisfies the open set condition (OSC).

For any $\epsilon \geq 0$, we say that the family $\left\{\left(S_{\sigma * 1}, \ldots, S_{\sigma * N}\right), \sigma \in D\right\}$ satisfies the finite intersection property of level $\epsilon$ (in short, $\epsilon$-FIP) if there exists a nonempty compact set $J$ in $E$ with $\operatorname{diam}(J)=\theta>0, \overline{J^{0}}=J$, and a number $e>1$ such that for $P$-a.e. $\omega$,

(iii) $J_{(|\sigma|+1, \sigma * i)} \subset J_{(|\sigma|, \sigma)}$ for all $i=1,2, \ldots, N$ and $\sigma \in D$. 
(iv) There exists $\delta \hat{=} \delta(\epsilon)>0$, for every $M \subset J$ with $\operatorname{diam}(M)<\min \{r, \delta\}$, we have

$$
\begin{aligned}
\operatorname{Card} H_{M}^{\omega} & \hat{=} \operatorname{Card}\left\{\sigma^{\omega} \in D: r^{k+1} \theta<\operatorname{diam}\left(J_{(|\sigma|, \sigma)}\right) \leq r^{k} \theta, J_{(|\sigma|, \sigma)} \cap M \neq \varnothing\right\} \\
& <e(\operatorname{diam} M)^{-\epsilon},
\end{aligned}
$$

where $J_{(|\sigma|, \sigma)}=S_{(|\sigma|, \sigma)}(J), J_{(0, \varnothing)}=J, k=\max \left\{n: \operatorname{diam}(M) \leq r^{n} \theta\right\}$.

REMARK 2.2. It is clear that if $0 \leq \epsilon_{1}<\epsilon_{2}$ then $\epsilon_{1}$-FIP implies $\epsilon_{2}$-FIP.

REMARK 2.3. If in the definition we have

$$
\limsup _{\operatorname{diam}(M) \rightarrow 0} \frac{\operatorname{Card} H_{M}}{k}<\infty
$$

then, for all $\epsilon>0$, the family $\left\{\left(S_{\sigma * 1}, \ldots, S_{\sigma * N}\right), \sigma \in D\right\}$ satisfies the $\epsilon$-FIP. Furthermore, if

$$
0<\limsup _{\operatorname{diam}(M) \rightarrow 0} \frac{\operatorname{Card} H_{M}}{k}<\infty
$$

exists, then the family $\left\{\left(S_{\sigma * 1}, \ldots, S_{\sigma * N}\right), \sigma \in D\right\}$ does not satisfy 0-FIP.

For simplicity, we write FIP for 0-FIP. Note that if $\left\{\left(S_{\sigma * 1}, \ldots, S_{\sigma * N}\right), \sigma \in D\right\}$ is i.i.d., then $K(\omega)$ is a statistically self-similar set (cf. [7, 8]). However, if $\left\{\left(r_{\sigma * 1}, \ldots, r_{\sigma * N}\right)\right.$, $\sigma \in D\}$ is i.i.d., then $K(\omega)$ need not be a statistically self-similar set [2].

LEMMA 2.4. If the family of $\left\{\left(S_{\sigma * 1}, \ldots, S_{\sigma * N}\right), \sigma \in D\right\} \subset \operatorname{sicon}(\Omega, E)^{N}$ satisfies the OSC, then it satisfies the FIP.

Proof. Suppose that $\left\{\left(S_{\sigma * 1}, \ldots, S_{\sigma * N}\right), \sigma \in D\right\}$ satisfies the OSC, and $O$ is defined as in (i) and (ii) in Definition 2.1. Take $J=\bar{O}$, since $S_{\tau}(O) \subset O$ for every $\tau \in D$, then

$$
\begin{aligned}
J_{(|\sigma|+1, \sigma * i)} & =S_{(|\sigma|, \sigma)}\left(S_{\sigma * i}(J)\right)=S_{(|\sigma|, \sigma)}\left(\overline{S_{\sigma * i}(O)}\right) \\
& \subset S_{(|\sigma|, \sigma)}(\bar{O})=J_{(|\sigma|, \sigma)},
\end{aligned}
$$

this means that (iii) is true.

Without loss of generality, we always assume that $\operatorname{diam}(J)=1$. Let $M \subset J$, $\operatorname{diam}(M)<$ $r$, by the definition of $k$, we have $\operatorname{diam}(M) \leq r^{k}$. For any $\sigma \in H_{M}$, we have

$$
\operatorname{diam}\left(J_{(|\sigma|, \sigma)}\right) \leq r^{k}, \quad J_{(|\sigma|, \sigma)} \cap M \neq \varnothing .
$$

Hence, there is a closed ball $B\left(x, 2 r^{k}\right)$ with center $x$ in $M$ and radius $2 r^{k}$ such that

$$
\bigcup_{\sigma \in H_{M}} J_{(|\sigma|, \sigma)} \subset B\left(x, 2 r^{k}\right) .
$$

Now we want to show that, for any $\sigma, \tau \in H_{M}$ with $\sigma \neq \tau, J_{(|\tau|, \tau)}^{0} \cap J_{(|\sigma|, \sigma)}^{0}=\varnothing$. Note that, by the definition of $H_{M}$, if $\sigma, \tau \in H_{M}, \sigma \neq \tau$, then $\sigma$ and $\tau$ are incomparable, that is, neither $\sigma|| \tau \mid=\tau$ nor $\tau|| \sigma \mid=\sigma$ holds. 
Write $\sigma \wedge \tau$ being the longest sequence $\gamma \in D$ such that $\gamma=\sigma|t=\tau| t$ for some integer $k$. Let $\sigma=\left(\sigma_{1}, \ldots, \sigma_{n}\right), \tau=\left(\tau_{1}, \ldots, \tau_{m}\right), l=|\sigma \wedge \tau|<\min \{n, m\}$, then

$$
\begin{aligned}
J_{(|\sigma|, \sigma)}^{0} \cap J_{(|\tau|, \tau)}^{0} & =S_{(l, \sigma \mid l)}\left(\left[S_{\sigma \mid(l+1)} \circ \cdots \circ S_{\sigma \mid n}(O)\right] \cap\left[S_{\tau \mid(l+1)} \circ \cdots \circ S_{\tau \mid m}(O)\right]\right) \\
& \subset S_{(l, \sigma \mid l)}\left(S_{\sigma \mid(l+1)}(O) \cap S_{\tau \mid(l+1)}(O)\right)=\varnothing .
\end{aligned}
$$

Then, by

$$
\begin{aligned}
\operatorname{diam}\left(J_{(|\sigma|, \sigma)}\right) & =\operatorname{diam}\left(S_{(|\sigma|, \sigma)}(J)\right)=\operatorname{Lip}\left(S_{(|\sigma|, \sigma)}\right), \\
\mathscr{L}\left(J_{(|\sigma|, \sigma)}\right) & =\operatorname{Lip}\left(S_{(|\sigma|, \sigma)}\right)^{d} \mathscr{L}(J),
\end{aligned}
$$

we have

$$
\mathscr{L}\left(\bigcup_{\sigma \in H_{M}} J_{(|\sigma|, \sigma)}\right) \geq \mathscr{L}\left(\bigcup_{\sigma \in H_{M}} J_{(|\sigma|, \sigma)}^{0}\right)=\sum_{\sigma \in H_{M}} \mathscr{L}\left(J_{(|\sigma|, \sigma)}\right) \geq\left(\operatorname{Card} H_{M}\right)\left(r^{k+1}\right)^{d} \mathscr{L}(J) .
$$

But

$$
\mathscr{L}\left(\bigcup_{\sigma \in H_{M}} J_{(|\sigma|, \sigma)}\right) \leq \mathscr{L}\left(B\left(x, 2 r^{k}\right)\right) \leq\left(2 r^{k}\right)^{d} V_{d},
$$

where $\mathscr{L}$ is the Lebesgue measure on $\mathbb{R}^{d}$ and $V_{d}$ is the Lebesgue measure of the unit ball in $\mathbb{R}^{d}$. So,

$$
\operatorname{Card} H_{M} \leq \frac{\left(2 r^{k}\right)^{d} V_{d}}{\left(r^{k+1}\right)^{d} \mathscr{L}(J)}
$$

and Lemma 2.4 is proved.

3. Main results. We always assume that $\left\{\left(S_{\sigma * 1}, \ldots, S_{\sigma * N}\right), \sigma \in D\right\} \subset \operatorname{sicon}(\Omega, E)^{\mathcal{N}}$ and let $\mathcal{N}=\{1,2, \ldots\}$ be the set of positive integers in this section. For almost every $\omega \in \Omega$, for any fixed positive integer $i \in \mathcal{N}$, we define a stopping $\gamma_{i}^{\omega}: C \mapsto \mathcal{N}$ by assigning to each $\sigma \in C$ the value

$$
\gamma_{i}^{\omega}(\sigma)=\min \left\{n: r_{\sigma \mid 1} \cdot r_{\sigma \mid 2} \cdots r_{\sigma \mid n} \leq r^{i}\right\}=\left\{n: \operatorname{Lip}\left(S_{(n, \sigma \mid n)}\right) \leq r^{i}\right\},
$$

and let

$$
\Gamma_{i}(\omega)=\left\{\bar{\sigma}_{i} \hat{=} \sigma \mid \gamma_{i}^{\omega}(\sigma): \sigma \in C\right\}
$$

then for $\sigma \in C$, we have $r^{i+1}<\operatorname{diam}\left(J_{\left(\left|\bar{\sigma}_{i}\right|, \bar{\sigma}_{i}\right)}\right)=\operatorname{Lip}\left(S_{\left(\left|\bar{\sigma}_{i}\right|, \bar{\sigma}_{i}\right)}\right) \leq r^{i}$. Note that for every $i \in\{1,2, \ldots\}$, we have

$$
\mathbf{E}\left(\sum_{\sigma \in \Gamma_{i}} r_{(\sigma,|\sigma|)}^{\alpha}\right)=1
$$


Proposition 3.1. If $\left\{\left(S_{\sigma * 1}, \ldots, S_{\sigma * N}\right), \sigma \in D\right\} \subset \operatorname{sicon}\left(\Omega, \mathbb{R}^{d}\right)^{N}$ satisfies condition (i) in Definition 2.1, and $\left\{\left(r_{\sigma * 1}, \ldots, r_{\sigma * N}\right), \sigma \in D\right\}$ is a family of i.i.d. random elements, then

$$
\operatorname{Dim} K(\omega) \leq \min \{\alpha, d\} \quad \text { a.e., }
$$

where $K(\omega)=\bigcap_{n=1}^{\infty} \bigcup_{\sigma \in C_{n}} S_{n, \sigma}(E)$, and $\alpha$ is the unique solution of (1.1).

Proof. Let $J$ be the nonempty compact subset of $E$ such that $\left\{\left(S_{\sigma * 1}, \ldots, S_{\sigma * N}\right)\right.$, $\sigma \in D\}$ is of FIP. First, we show that

$$
K(\omega)=\bigcap_{n=1}^{\infty} \bigcup_{\sigma \in C_{n}} S_{n, \sigma}(J) .
$$

In fact, for $\sigma \in C, \lim _{n \rightarrow \infty} S_{n, \sigma \mid n}(E)=\left\{x_{\sigma}\right\}$ is a singleton, and $\lim _{n \rightarrow \infty} S_{n, \sigma \mid n}(J)=\left\{y_{\sigma}\right\}$ is a singleton also, and since $J \subset E$, we have $x_{\sigma}=y_{\sigma}$, so (3.5) holds.

Define a metric $\rho^{*}(\sigma, \tau) \hat{=} r^{(|\sigma \wedge \tau|)}$, then $\left(C, \rho^{*}\right)$ is a complete, separable, compact, and totally disconnected metric space [5]. A random code map $\pi^{\omega}: C \mapsto K(\omega)$ is defined by $\pi(\sigma)=x_{\sigma}$, then for $P$-a.e. $\omega \pi$ is continuous and $\pi(C)=K$.

For all $\delta \in(0, r), k_{0} \hat{=} \min \left\{k: r^{k+1}<2 \delta\right\}$, let $\left\{B\left(x_{i}, \delta\right), i=1,2, \ldots, N(\delta)\right\}$ be a collection of balls with center $x$ in $K$ and radius $\delta$ (note that $N(\delta)$ is finite), then there exist $\sigma\langle i\rangle \in C$, such that $x_{i}=\pi(\sigma\langle i\rangle), i=1,2, \ldots$ Note that $\left\{B\left(x_{i}, \delta\right)\right\}$ are disjoint. For each

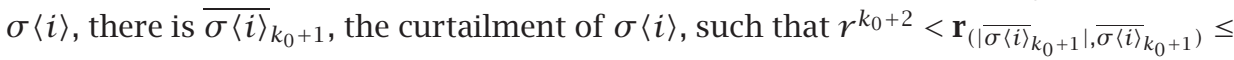
$r^{k_{0}+1}$. If $x_{i} \neq x_{j}$, then $\overline{\sigma\langle i\rangle}_{k_{0}+1} \neq \overline{\sigma\langle j\rangle}_{k_{0}+1}$, otherwise, if ${\overline{\sigma\langle i\rangle_{k_{0}+1}}}=\overline{\sigma\langle j\rangle}_{k_{0}+1}$ we have

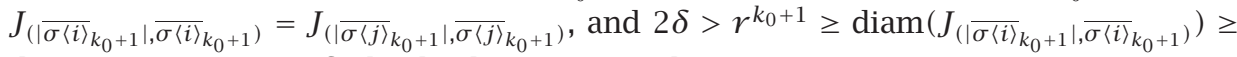
$\operatorname{dist}(\pi(\sigma), \pi(\sigma))=2 \delta$, this leads to a contradiction.

Hence, we have

$$
\begin{aligned}
\mathbf{E}\left(N(\delta) r^{\left(k_{0}+2\right)(\alpha+\epsilon)}\right) & \leq \mathbf{E}\left(\sum_{i} \operatorname{diam}\left(B\left(x_{i}, \delta\right)\right)^{\alpha+\epsilon} r^{\alpha+\epsilon}\right) \\
& \leq \mathbf{E}\left(\sum_{\overline{\sigma\langle i\rangle_{k_{0}+1}}} \in \Gamma_{k_{0}+1} \mathbf{r}_{\left(|\overline{\sigma\langle i}\rangle_{k_{0}+1} \mid, \overline{\sigma\langle i\rangle_{k_{0}+1}}\right.}^{\alpha+\epsilon}\right) \\
& \leq \sum_{k=1}^{\infty} \mathbf{E}\left(\sum_{i=1}^{N} r_{i}^{\alpha+\epsilon}\right)^{k}<\infty
\end{aligned}
$$

thus for $P$-a.e. $\omega$,

$$
\limsup _{\delta \rightarrow 0} \frac{\log N(\delta)}{-\log \delta} \leq \limsup _{\delta \rightarrow 0} \frac{\log r^{\left(k_{0}+2\right) \alpha}}{\log \delta} \leq \alpha+\epsilon
$$

we obtain that, for almost all $\omega$, $\operatorname{Dim} K \leq \alpha+\epsilon$ for arbitrary $\epsilon>0$, which means that $\operatorname{Dim} K \leq \min \{d, \alpha\}$ a.e.

LEMmA 3.2. Let $T_{n}=\sum_{\sigma \in C_{n}} \mathbf{r}_{(n, \sigma)}^{\alpha}$, then $Y=\lim _{n \rightarrow \infty} T_{n}$ exists a.e. and $\mathbf{E}(Y)=1$, $\infty>Y>0$ a.e. 
Proof. Let $\mathscr{F}_{n} \hat{=} \sigma\left\{r_{\sigma},|\sigma| \leq n\right\}$, then $\left\{T_{n}, \mathscr{F}_{n}\right\}$ is a martingale, and $\mathbf{E} T_{n}=1$ for $n=1,2, \ldots$, by the martingale convergence theorem, $Y<\infty$ a.e. exists, and $\mathbf{E} Y=1$.

The equation $Y_{\sigma} \hat{=} \lim _{n \rightarrow \infty} \sum_{\tau \in C_{n}}\left(\prod_{t=1}^{n} r_{\sigma *(\tau \mid t)}^{\alpha}\right)$ exists for all $\sigma \in D$ by the similar argument as above (cf. [1, 12]), and $\left\{Y_{\sigma}, \sigma \in D\right\}$ have the same distributions as that of $Y$ by the i.i.d. property of $\left\{\left(r_{\sigma * 1}, \ldots, r_{\sigma * N}\right), \sigma \in D\right\}$ and $\left\{Y_{\sigma}, \sigma \in C_{n}\right\}$ is an i.i.d. family. Note that

$$
Y=\sum_{\sigma \in C_{n}} \mathbf{r}_{(n, \sigma)}^{\alpha} Y_{\sigma} \quad \text { a.e. }
$$

If $P\{Y=0\}=a$, then $a=P\left\{Y=\sum_{i=1}^{N} r_{i}^{\alpha} Y_{i}=0\right\}=P\left\{Y_{i}=0\right\}^{N}=a^{N}$, then $a=0$ or $a=1$. Since $\mathbf{E} Y=1$, we have $a=0$. The lemma is proved.

LEMMA 3.3. For P-a.e. $\omega$, there is a random measure $v^{\omega}$ on $C$ satisfying

$$
v([\sigma])=\mathbf{r}_{(|\sigma|, \sigma)}^{\alpha} Y_{\sigma}, \quad v(C)=Y .
$$

Proof. By the definition of the metric $\rho^{*}$, the cylinder $[\sigma]$ is both open and closed, since

$$
[\sigma]=\left\{\tau \in C: \rho(\tau, \sigma) \leq r^{|\sigma|}\right\}=\left\{\tau \in C: \rho(\tau, \sigma)<r^{|\sigma|-1}\right\}
$$

for every $\sigma \in D$. Let $\mathscr{B}$ be the collection of all cylinders $[\sigma], \sigma \in D$, and let $\mathscr{A}$ be the collection of a finite union of disjoint cylinders, where by convention the empty union is taken to be the empty set $\varnothing$. Then $\mathscr{A}$ is an algebra. Define a random set function $\nu^{\omega}$ by $v(\varnothing)=0, v([\sigma]) \hat{=} \mathbf{r}_{(|\sigma|, \sigma)}^{\alpha} Y_{\sigma}$, for $\sigma \in D$, then by (3.8) we have that

$$
v([\sigma])=\sum_{i=1}^{N} v([\sigma * i]), \quad v(C)=Y \quad \text { a.e., }
$$

so for almost every $\omega$, the set function $v$ is well defined. By the compactness of $C$, it can be easily seen that if $A_{n} \in \mathscr{A}$ decreases to $\varnothing$, then $A_{n}=\varnothing$ for $n$ large enough, so that $v\left(A_{n}\right)$ decreases to $\varnothing$; this shows that $v$ is a measure on $\mathscr{A}$. In a natural way, we can extend $v$ to a Borel measure on $\sigma(\mathscr{B})$ (cf. [11]).

Proposition 3.4. If for every $\epsilon>0,\left\{\left(S_{\sigma * 1}, \ldots, S_{\sigma * N}\right), \sigma \in D\right\} \subset \operatorname{sicon}\left(\Omega, \mathbb{R}^{d}\right)^{N}$ satisfying the $\epsilon$-FIP, $\left\{\left(r_{\sigma * 1}, \ldots, r_{\sigma * N}\right), \sigma \in D\right\}$ is a family of i.i.d. random elements, then $\operatorname{dim}(K) \geq \min \{\alpha, d\}$ a.e.

Proof. Let $\mu^{\omega \hat{=}} \nu^{\omega} \circ \pi^{-1}$, then $\mu$ is a random measure and $\operatorname{supp}(\mu)=K, \mu(K)=$ $v(C)=Y>0$ a.e. By Proposition 3.1, we have $\operatorname{dim}(K) \leq d$. For $\alpha \leq d$, if we can prove that for every $\epsilon>0$, there exists $r>\delta>0$ and a random variable $\infty>l_{1}(\omega)>0$ a.e. such that if $M \subset J$ and $\operatorname{diam}(M)<\delta$, then $\mu(M) \leq l_{1} \operatorname{diam}(M)^{\alpha-2 \epsilon}$ a.e., then it follows from the mass distribution principle, (cf. Falconer [4]), $\operatorname{dim} K \geq \alpha$ a.e. 
In fact,

$$
\begin{aligned}
\mu(M) & =\mu(M \cap K)=v \circ \pi^{-1}(M \cap K) \leq v \circ \pi^{-1}\left(\bigcup_{\sigma \in H_{M}} J_{(|\sigma|, \sigma)}\right) \\
& \leq e(\operatorname{diam} M)^{-\epsilon} \max _{\sigma \in H_{M}, \sigma \in D} v \circ \pi^{-1}\left(J_{(|\sigma|, \sigma)}\right) \\
& =e(\operatorname{diam} M)^{-\epsilon} \cdot v\left\{\tau \in C: \pi(\tau) \in J_{\left(\left|\sigma_{o}\right|, \sigma_{0}\right)}\right\}
\end{aligned}
$$

by the $\epsilon$-FIP, where $v\left(J_{\left(\left|\sigma_{0}\right|, \sigma_{o}\right)}\right)=\max \left\{v\left(J_{(|\gamma|, \gamma)}\right), \gamma \in H_{M}\right\}$. If $\pi(\tau) \in J_{\left(\left|\sigma_{0}\right|, \sigma_{o}\right)}$, then $J_{\left(\left|\bar{\tau}_{k}\right|, \bar{\tau}_{k}\right)} \cap J_{\left(\left|\sigma_{0}\right|, \sigma_{0}\right)} \neq \varnothing$ and $r^{k+1}<\operatorname{diam}\left(J_{\left(\left|\bar{\tau}_{k}\right|, \bar{\tau}_{k}\right)}\right) \leq r^{k}$, so $\bar{\tau}_{k} \in H_{J_{\left(\left|\sigma_{0}\right|, \sigma_{0}\right)}}$, and by $\epsilon$-FIP again, there are at most $\left[e(\operatorname{diam} M)^{-\epsilon}\right]+1$ such $\bar{\tau}_{k}^{1}, \ldots, \bar{\tau}_{k}^{\left[e \cdot(\operatorname{diam} M)^{-\epsilon}\right]+1}$ satisfying the above property, so

$$
\left\{\boldsymbol{\tau} \in C: \pi(\boldsymbol{\tau}) \in J_{\left(\left|\sigma_{0}\right|, \sigma_{o}\right)}\right\} \subset\left[\overline{\boldsymbol{\tau}}_{k}^{1}\right] \cup \cdots \cup\left[\overline{\boldsymbol{\tau}}_{k}^{\left[e \cdot(\operatorname{diam} M)^{-\epsilon}\right]+1}\right],
$$

hence

$$
\begin{aligned}
\mu(M) & \leq 2 e^{2}(\operatorname{diam} M)^{-2 \epsilon} \max _{1 \leq i \leq\left[e(\operatorname{diam} M)^{-\epsilon}\right]+1} v\left[\bar{\tau}_{k}^{i}\right] \\
& =2 e^{2}(\operatorname{diam} M)^{-2 \epsilon} \max _{i=1, \ldots,\left[e(\operatorname{diam} M)^{-\epsilon}\right]+1} r_{\left(\left|\bar{\tau}_{k}^{i}\right|, \bar{\tau}_{k}^{i}\right)}^{\alpha} Y_{\tilde{\tau}_{k}^{i}} .
\end{aligned}
$$

Since $\bar{\tau}_{k}^{i} \in H_{J_{\left(\left|\sigma_{0}\right|, \sigma_{0}\right)}}, \sigma_{0} \in H_{M}$, we have $r^{k+1}<\mathbf{r}_{\left(\left|\tilde{\tau}_{k}^{i}\right|, \tilde{\tau}_{k}^{i}\right)} \leq r^{k}$, therefore,

$$
\mu(M) \leq 2 e^{2} \max _{1 \leq i \leq\left[e(\operatorname{diam} M)^{-\epsilon}\right]+1} \frac{Y_{\bar{\tau}_{k}^{i}} \operatorname{diam}(M)^{\alpha-2 \epsilon}}{r^{\alpha}}
$$

take $l_{1}=2 e^{2} \max _{1 \leq i \leq\left[e(\operatorname{diam} M)^{-\epsilon}\right]+1} Y_{\bar{\tau}_{k}^{i}} / \gamma^{\alpha}$, note that $\left\{Y_{\sigma}, \sigma \in D\right\}$ have the same distribution as $Y$, so $0<l_{1}<\infty$ a.e., hence we have $\mu(M)<l_{1} \operatorname{diam}(M)^{\alpha-2 \epsilon}<\infty$ a.e. The proposition is proved.

Proposition 3.5. If $\left\{\left(S_{\sigma * 1}, \ldots, S_{\sigma * N}\right), \sigma \in D\right\} \subset \operatorname{sicon}\left(\Omega, \mathbb{R}^{d}\right)^{N}$ satisfies FIP, $\left\{\left(r_{\sigma * 1}, \ldots, r_{\sigma * N}\right), \sigma \in D\right\}$ is a family of i.i.d. random elements, and furthermore $\sum_{i=1}^{N} r_{i}^{\alpha}=1$ a.e. and $\alpha \leq d$, where $\alpha$ is defined in (1.1), then $\infty>\mathscr{H}^{\alpha}(K)>0$ a.e.

Proof. Since $\left\{J_{(n, \sigma)}, \sigma \in C_{n}\right\}$ is a covering of $K$ for almost all $\omega \in \Omega$, and $\mathbf{r}_{(n, \sigma)} \rightarrow 0$, we have

$$
\mathbf{E}\left(\mathscr{H}^{\alpha}(K)\right) \leq \mathbf{E}\left(\sum_{\sigma \in C_{n}} \mathbf{r}_{(n, \sigma)}^{\alpha}\right)=1<\infty
$$

so $\mathscr{H}^{\alpha}(K)<\infty$ a.e.

Suppose that $\sum_{i=1}^{N} r_{i}^{\alpha}=1$ a.e., then $Y=1$ a.e. by its definition. If $\mathscr{H}^{\alpha}(K)<1 / l_{1}$ a.e., then there would be a collection $\mathscr{E}$ of sets each with diameter less than $r$ and covering $K$ such that $\sum_{E \in \mathscr{E}} \operatorname{diam}(E)^{\alpha}<1 / l_{1}$ a.e. But taking $\epsilon=0$ in (3.15),

$$
1>\sum_{E \in \mathscr{E}} l_{1} \operatorname{diam}(E)^{\alpha} \geq \sum_{E \in \mathscr{E}} \mu(E) \geq \mu(K)=1 \quad \text { a.e. }
$$

This would lead to a contradiction. So this proposition holds. 
Combining Propositions 3.1, 3.4, and 3.5, we obtain Theorem 1.1.

COROLLARY 3.6. In the deterministic case with specific maps $\left\{S_{1}, \ldots, S_{N}\right\}$, FIP is equal to the open set condition.

Proof. By Proposition 3.5, $\infty>\mathscr{H}^{\alpha}(K)>0$, where $\alpha$ is the unique solution of $\sum_{i=1}^{N} \operatorname{Lip}\left(S_{i}\right)^{\alpha}=1$. Combining with Schief's result [13], the corollary is true.

Corollary 3.7. If $\left\{\left(S_{\sigma * 1}, \ldots, S_{\sigma * N}\right), \sigma \in D\right\} \subset \operatorname{sicon}\left(\Omega, \mathbb{R}^{d}\right)^{N}$ satisfies the FIP, $\left\{\left(r_{\sigma * 1}, \ldots, r_{\sigma * N}\right), \sigma \in D\right\}$ is a family of i.i.d. random elements, then $\operatorname{dim} \mu=$ $\operatorname{Dim} \mu=\alpha$ a.e., where $\mu=v \circ \pi^{-1}$.

Proof. For every $B(x, h)$ with center $x \in K$ and radius $h<r$, we have

$$
\mu(B(x, h)) \leq l_{1} h^{\alpha}
$$

by (3.15), hence

$$
\liminf _{h \rightarrow 0} \frac{\ln \mu(B(x, h))}{\ln h} \geq \alpha \quad \text { a.e. }
$$

On the other hand, there exists $\sigma \in C$, such that $\pi(\sigma)=x$, take $\bar{\sigma}$, the curtailment of $\sigma$, such that $(1 / 2) r^{k+1}<\left|J_{\left(|\bar{\sigma}|, \bar{\sigma}_{k}\right)}\right| \leq(1 / 2) r^{k}$, where $k=\max \left\{n: h \leq r^{n}\right\}$, so $J_{(|\bar{\sigma}|, \bar{\sigma})} \subset$ $B$, we have

$$
\mu(B(x, h))=v \circ \pi^{-1}(B(x, h)) \geq v \circ \pi^{-1}\left(J_{(\bar{\sigma}, \bar{\sigma})}\right) \geq v[\bar{\sigma}]=r_{(|\bar{\sigma}|, \bar{\sigma})}^{\alpha} Y_{\bar{\sigma}}
$$

thus

$$
\lim _{h \rightarrow 0} \frac{\ln \mu(B(x, h))}{\ln h} \leq \alpha \quad \text { a.e. }
$$

Therefore, $\operatorname{dim} \mu=\operatorname{Dim} \mu=\alpha$ a.e.

4. Examples. First, we give an example which satisfies the FIP but not the OSC.

EXAMPLE 4.1. Let $J=[0,1]$, and let $N=2, r_{\sigma * 1}=1 / 3, r_{\sigma * 2}=1 / 27, \sigma \in D$.

(I) The first two steps:

$$
\begin{gathered}
s_{1}^{1}(x)=\frac{1}{3} x, \quad s_{2}^{1}(x)=\frac{1}{27} x+\frac{8}{27} \\
s_{11}^{1}(x)=\frac{1}{9} x, s_{12}^{1}(x)=\frac{1}{81} x+\frac{2}{9}, \quad s_{21}^{1}(x)=\frac{1}{81} x+\frac{8}{27}, \\
s_{22}^{1}(x)=\frac{1}{27 \times 27} x+\left(\frac{1}{3}-\frac{1}{27 \times 27}\right) .
\end{gathered}
$$

Thus we have four basic intervals.

(II) The second two steps: as to the interval $[0,1 / 9]$, we repeat the same construction technique as in (I). As to the rest of the three basic intervals, we can easily find maps with ratios $\left\{r_{\sigma * 1}, r_{\sigma * 2}\right\}=\{1 / 3,1 / 27\}$ for $\sigma \in C_{3}$ and $C_{4}$, such that the subsets of all these three basic intervals are disjoint. And so on. 
Proof. Obviously, we cannot find an open set such that $\left\{\left(S_{\sigma * 1}, S_{\sigma * 2}\right), \sigma \in D\right\}$ satisfies OSC, but FIP holds on it. By our theorem, $\operatorname{dim}(K)=\operatorname{Dim}(K)=\alpha$, where $\alpha$ is the solution of $(1 / 3)^{\alpha}+(1 / 27)^{\alpha}=1$.

Then we give an example which satisfies the $\epsilon$-FIP for every $\epsilon>0$, but does not satisfy the FIP.

EXAMPLE 4.2. Let $J=[0,1]$, and let $S_{1}(x)=r x, S_{2}(x)=r x+(1-r)$ for $1 / 2<r<1$, then $\left\{S_{1}, S_{2}\right\}$ does not satisfy the OSC. But if $r=\left(5^{1 / 2}-1\right) / 2$ is a PV number, then $\left\{\left(S_{\sigma * 1}, S_{\sigma * 2}\right), \alpha \in D\right\}$ satisfies the FIP, where $D=\bigcup_{n=1}^{\infty}\{1,2\}^{n}$.

Proof. By Lau's and Nagi's result in [10], if $r$ is a PV number, then $\left\{S_{1}, S_{2}\right\}$ satisfies the weak separation property, that is, there exist $z_{0} \in J$ and a positive integer $l$ such that, for any $z=S_{(\tau,|\tau|)}\left(z_{0}\right)$, every closed $r^{k}$-ball contain at most $l$ distinct $S_{(|\sigma|, \sigma)}(z)$, $\sigma \in \Gamma_{k},\left(S_{(|\sigma|, \sigma)}(z)\right.$ can be repeated, that is, we allow that $S_{(\sigma,|\sigma|)}(z)=S_{\left(\sigma^{\prime},\left|\sigma^{\prime}\right|\right)}(z)$ for $\left.\sigma \neq \sigma^{\prime}, \sigma, \sigma^{\prime} \in \Gamma_{k}\right)$.

Denote $J_{(|\sigma|, \sigma)}=S_{(|\sigma|, \sigma)}(J)$ for $\sigma \in D$, Definition 2.1(iii) holds obviously. Without loss of generality, let $M$ be an interval in $J$ with $\operatorname{diam}(M)<\delta$ ( $\delta$ will be defined in the following proof), $r^{k+1}<\operatorname{diam}(M) \leq r^{k}, H_{M}$ is the set given in Definition 2.1, let $B=B\left(x, 2 r^{k}\right)$ be an interval with center $x \in M$ and radius $2 r^{k}$, then $\bigcup_{\sigma \in H_{M}} J_{(|\sigma|, \sigma)} \subset$ $B$ and $M \subset B$. According to [10, Remark 3], "under the weak separation property, every $h r^{k}$-ball with $h>0$ contains at most $l[2 h]^{d}$ distinct $S_{(|\sigma|, \sigma)}(z), \sigma \in \Gamma_{k}$," for $z_{0} \in J$ and $\sigma \in H_{M}$, there are at most $4 l$ distinct $S_{(|\sigma|, \sigma)}\left(z_{0}\right) \in B \sigma \in H_{M} \subset \Gamma_{k}$ (note that $\Gamma_{k}=C_{k}$ in this example). If $\sigma\langle 1\rangle \neq \sigma\langle 2\rangle \neq \cdots \in H_{M}$, but $S_{(|\sigma\langle 1\rangle|, \sigma\langle 1\rangle)}\left(z_{0}\right)=$ $S_{(|\sigma\langle 2\rangle|, \sigma\langle 2\rangle)}\left(z_{0}\right)=\cdots$, then for $z_{1} \neq z_{0}, z_{1}=S_{(|\tau|, \tau)}\left(z_{0}\right)$ for some $\tau \in D$, we have at most $4 l$ distinct $S_{(|\sigma\langle 1\rangle|, \sigma\langle 1\rangle)}\left(z_{1}\right), S_{(|\sigma\langle 2\rangle|, \sigma\langle 2\rangle)}\left(z_{1}\right), \ldots, S_{(|\sigma\langle 4 l\rangle|, \sigma\langle 4 l\rangle)} \in B$ for $\sigma\langle j\rangle \in \Gamma_{k}$.

For a fixed $j \in\{1,2, \ldots, 4 l\}$, if

$$
S_{(|\sigma\langle j 1\rangle|, \sigma\langle j 1\rangle)}\left(z_{1}\right)=S_{(|\sigma\langle j 2\rangle|, \sigma\langle j 2\rangle)}\left(z_{1}\right)=\cdots=S_{(|\sigma\langle j\rangle|, \sigma\langle j\rangle)}\left(z_{1}\right)
$$

for $\sigma\langle j i\rangle \in \Gamma_{k}$, we get

$$
S_{(|\sigma\langle j i\rangle|, \sigma\langle j i\rangle)}(x)=S_{(|\sigma\langle j\rangle|, \sigma\langle j\rangle)}(x), \quad \forall x \in[0,1] i=1,2, \ldots .
$$

By induction, we can get that

$$
N(k) \leq\left[\frac{k+1}{2}\right] \leq k
$$

for $j=1, \ldots, 4 l$, where $N(k)$ is the cardinality of $i$ which satisfies (4.3).

Thus, for every $\epsilon>0$, let $N_{0} \hat{=} \min \left\{k: k \leq r^{-k \epsilon}\right\}$, take $\delta \hat{=} \min \left\{r^{N_{0}}, r\right\}$, we have Card $H_{M} \leq(4 l)^{2}(\operatorname{diam}(M))^{-\epsilon}$. It is easy to show that $\operatorname{dim} K=\operatorname{Dim} K=1$.

EXAMPLE 4.3. Let $(\Omega, \mathscr{F}, P)$ be $((0,1], \mathscr{B}(0,1], \mathscr{L})$. Take $J=[0,1]$, then we will construct a random recursive set, for $\omega \in \Omega$, by the following procedure:

(I) The first three steps:

(1) let

$$
S_{1}^{1}(x)=\frac{x}{3}, \quad S_{2}^{1}(x)=\frac{x}{3}+\frac{2}{3}
$$


(2) let

$$
\begin{aligned}
S_{11}^{1}(x) & =S_{1}^{1} \circ S_{1}^{1}(x)=\frac{x}{9}, \quad S_{12}^{1}(x)=\frac{x}{3^{2}}+\left(\left(\frac{1}{3}\right)^{2}-\left(\frac{1}{3}\right)^{3} \omega\right), \\
S_{21}^{1} & =S_{2}^{1} \circ S_{1}^{1}(x), \quad S_{22}^{1}=S_{2}^{1} \circ S_{2}^{1}(x) ;
\end{aligned}
$$

(3) let

$$
\begin{array}{ll}
S_{111}^{1}(x)=S_{1}^{1} \circ S_{1}^{1} \circ S_{1}^{1}(x), & S_{112}^{1}(x)=S_{1}^{1} \circ S_{1}^{1} \circ S_{2}^{1}(x)=\frac{x}{27}+\frac{2}{27}, \\
S_{121}^{1}(x)=S_{112}^{1}+\left(\frac{1}{3}\right)^{3}, \quad & S_{122}^{1}(x)=S_{112}^{1}+\left(\left(\frac{1}{3}\right)^{2}-\left(\frac{1}{3}\right)^{3} \omega\right), \\
S_{211}^{1}(x)=S_{2}^{1} \circ S_{1}^{1} \circ S_{1}^{1}(x), & S_{212}^{1}(x)=S_{2}^{1} \circ S_{1}^{1} \circ S_{2}^{1}(x), \\
S_{221}^{1}(x)=S_{2}^{1} \circ S_{2}^{1} \circ S_{1}^{1}(x), & S_{222}^{1}(x)=S_{2}^{1} \circ S_{2}^{1} \circ S_{2}^{1}(x) .
\end{array}
$$

(II) The second three steps. Replacing $[0,1]$ by $[0,1 / 27]$, we repeat the above iterative procedure:

(1) let

$$
S_{1}^{2}(x)=\frac{x / 27}{3}, \quad S_{2}^{2}(x)=\frac{x / 27}{3}+\frac{2 / 27}{3}
$$

(2) let

$$
\begin{array}{ll}
S_{11}^{2}(x)=S_{1}^{2} \circ S_{1}^{2}(x), & S_{12}^{2}(x)=\frac{x / 27}{3^{2}}+\left(\left(\frac{1}{3}\right)^{2+3}-\left(\frac{1}{3}\right)^{3+3} \omega\right), \\
S_{21}^{2}(x)=S_{2}^{2} \circ S_{1}^{2}(x), & S_{22}^{2}(x)=S_{2}^{2} \circ S_{2}^{2}(x) ;
\end{array}
$$

(3) let

$$
\begin{array}{ll}
S_{111}^{2}(x)=S_{1}^{2} \circ S_{1}^{2} \circ S_{1}^{2}(x), & S_{112}^{2}(x)=S_{1}^{2} \circ S_{1}^{2} \circ S_{2}^{2}(x)=\frac{x / 27}{27}+\frac{2 / 27}{27}, \\
S_{121}^{2}(x)=S_{112}^{2}+\left(\frac{1}{3}\right)^{3+3}, & S_{122}^{2}(x)=S_{112}^{2}+\left(\left(\frac{1}{3}\right)^{2+3}-\left(\frac{1}{3}\right)^{3+3} \omega\right), \\
S_{211}^{2}(x)=S_{2}^{2} \circ S_{1}^{2} \circ S_{1}^{2}(x), & S_{212}^{2}(x)=S_{2}^{2} \circ S_{1}^{2} \circ S_{2}^{2}(x), \\
S_{221}^{2}(x)=S_{2}^{2} \circ S_{2}^{2} \circ S_{1}^{2}(x), & S_{222}^{2}(x)=S_{2}^{2} \circ S_{2}^{2} \circ S_{2}^{2}(x) .
\end{array}
$$

(III) Replacing $[0,1 / 27]$ by $[0,1 / 27 \times 27]$, we repeat the same iterative procedure again. And so on.

It is not easy to find an open set $O$ such that $\left\{\left(S_{\sigma * 1}, \ldots, S_{\sigma * N}\right), \sigma \in D\right\}$ satisfies the OSC, but we can easily see that for every $\omega \in \Omega,\left\{\left(S_{\sigma * 1}, \ldots, S_{\sigma * N}\right), \sigma \in D\right\}$ satisfies the FIP, so according to Theorem 1.1, we obtain that $\operatorname{Dim} K=\operatorname{dim} K=\ln 2 / \ln 3$ a.e. and the Hausdorff measure of the random set $K(\omega)$ is finite and positive a.e. 
ACKNOWLEDGments. The authors thank Prof. Yimin Xiao for his suggestion, they also thank Dr. A. Deng and Dr. J. Yu for their helpful discussion. The research is supported by the National Natural Science Foundation of China, the Foundation of Wuhan University, and partially by the Foundation of Henan Normal University.

\section{REFERENCES}

[1] M. Arbeiter and N. Patzschke, Random self-similar multifractals, Math. Nachr. 181 (1996), 5-42.

[2] R. Cawley and R. D. Mauldin, Multifractal decompositions of Moran fractals, Adv. Math. 92 (1992), no. 2, 196-236.

[3] K. J. Falconer, Random fractals, Math. Proc. Cambridge Philos. Soc. 100 (1986), no. 3, 559-582.

[4] _ Fractal Geometry, John Wiley \& Sons, Chichester, 1990.

[5] K. J. Falconer and T. C. O'Neil, Vector-valued multifractal measures, Proc. Roy. Soc. London Ser. A 452 (1996), no. 1949, 1433-1457.

[6] S. Graf, Statistically self-similar fractals, Probab. Theory Related Fields 74 (1987), no. 3, 357-392.

[7] D. Hu, I.i.d. statistical contraction operators and statistically self-similar sets, to appear in Chinese Ann. of Math.

[8] - The necessary and sufficient conditions for various self-similar sets and their dimension, Stochastic Process. Appl. 90 (2000), no. 2, 243-262.

[9] J. E. Hutchinson, Fractals and self-similarity, Indiana Univ. Math. J. 30 (1981), no. 5, 713747.

[10] K.-S. Lau and S.-M. Ngai, Multifractal measures and a weak separation condition, Adv. Math. 141 (1999), no. 1, 45-96.

[11] Q. Liu, Exact packing measure on a Galton-Watson tree, Stochastic Process. Appl. 85 (2000), no. 1, 19-28.

[12] R. D. Mauldin and S. C. Williams, Random recursive constructions: asymptotic geometric and topological properties, Trans. Amer. Math. Soc. 295 (1986), no. 1, 325-346.

[13] A. Schief, Separation properties for self-similar sets, Proc. Amer. Math. Soc. 122 (1994), no. 1, 111-115.

Hongwen Guo: College of Mathematics and Statistics, Wuhan University, Wuhan 430072, CHINA

E-mail address: guo-hongwen@263. net

Dihe Hu: College of Mathematics AND STATistics, WuHAN UNiversity, WuHAN 430072, CHINA

E-mail address: dhhu@whu.edu.cn 


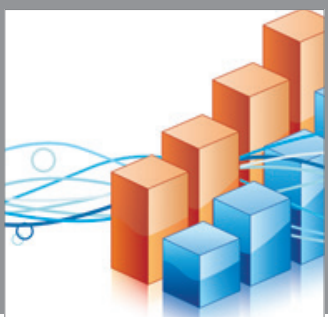

Advances in

Operations Research

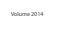

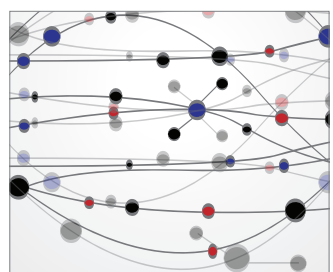

\section{The Scientific} World Journal
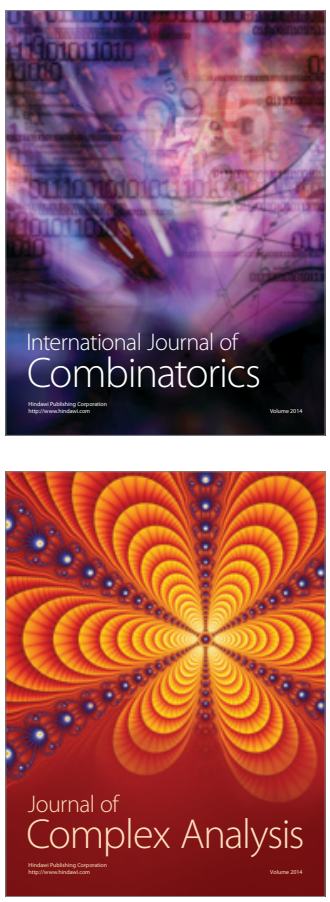

International Journal of

Mathematics and

Mathematical

Sciences
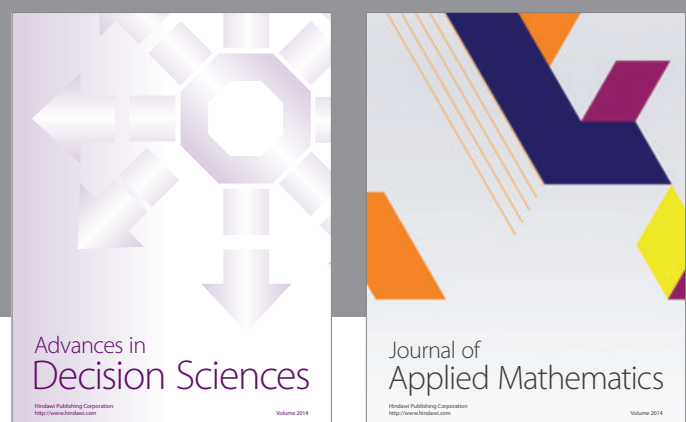

Journal of

Applied Mathematics
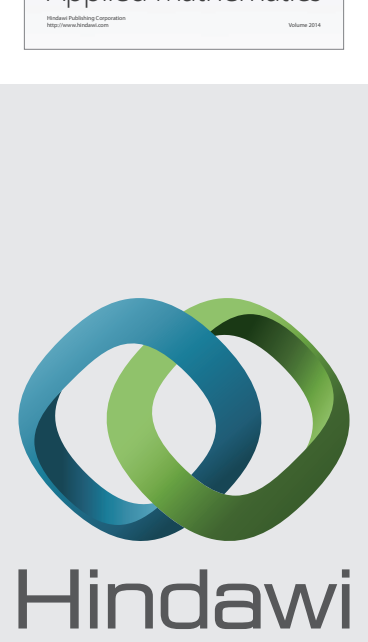

Submit your manuscripts at http://www.hindawi.com
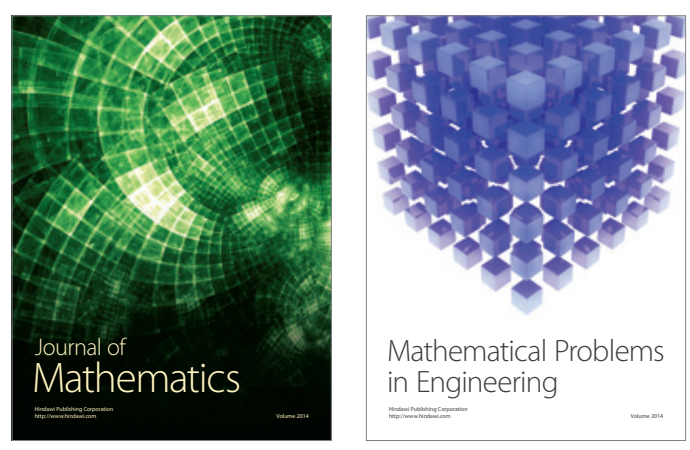

Mathematical Problems in Engineering
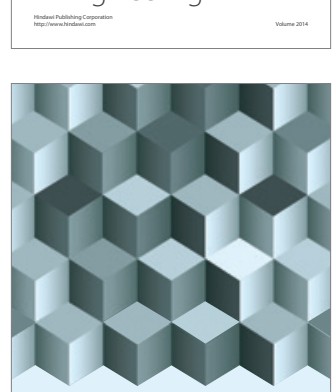

Journal of

Function Spaces
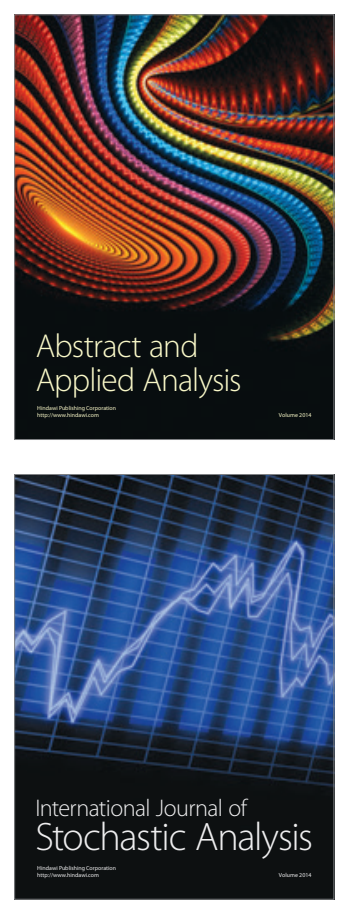

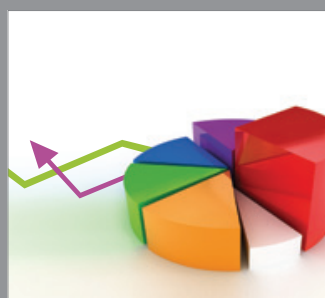

ournal of

Probability and Statistics

Promensencen
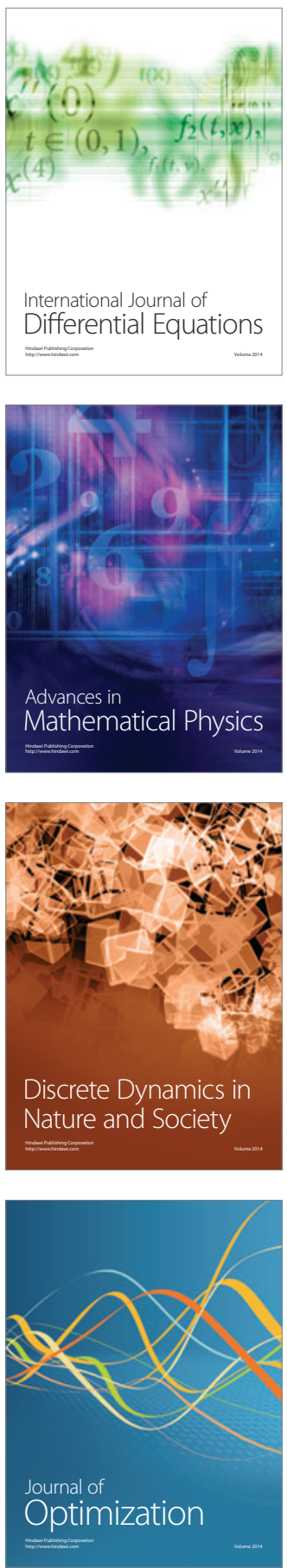